\title{
FACTORS IN THE RESISTANCE OF GONORRHEA TO SULFONAMIDES
}

\author{
By WALTER T. GOODALE AND LOUIS SCHWAB \\ (From the Department of Bacteriology and Immunology, Harvard Medical School and School of \\ Public Health, Boston)
}

(Received for publication August 12, 1943)

\section{INTRODUCTION}

The problem of resistance to sulfonamide therapy ${ }^{1}$ in male gonorrhea is well known. Cox (1) states that, in varying degrees, clinical resistance is found in approximately 25 per cent of cases treated with sulfadiazine or sulfathiazole. More recently, the incidence of resistance has risen to approximately 50 per cent of cases admitted to the Boston Dispensary (2). This resistance is manifested either by persistence of symptoms or by persistence of positive cultures in asymptomatic carriers. Since gonorrhea is usually a self-limited disease, Cox emphasizes the importance of considering all cases as drug failures which do not clear up within 2 weeks after starting sulfonamide therapy. It is likewise important to recognize relapse after apparent cure as a manifestation of resistance, which is often missed in the clinic unless patients are followed every few days by smear, culture, and symptomatology for at least 4 weeks.

Petro (3) summarizes the factors in sulfonamide resistance, emphasizing (1) factors interfering with the transport of drug to the site of infection in adequate dosage, (2) factors interfering with proper drainage of the products of inflammation, (3) factors within the invading organism, and (4) factors within the host and its bodily defenses.

Determination of factors within the gonococcus responsible for this clinical resistance is a logical initial approach. To this end, correlation of the clinical course with laboratory observations on the growth characteristics of the organisms isolated from individual cases in the presence of sulfonamides has been reported by Cohn et al. (4),

\footnotetext{
1 Sulfanilamide and sulfapyridine are no longer commonly used in the therapy of gonorrhea; in this paper, "sulfonamide therapy" refers to the use of sulfathiazole, sulfadiazine, or sulfamerizine therapy-these being the newer and more effective drugs.
}

Bang and Bang (5), Petro (3), and, most recently, by Lankford et al. (6), in a series of 200 female cases. All of these investigators report that organisms from clinically resistant cases show, by their various methods, an increased tolerance to sulfonamides in the laboratory. Harkness (7) reports a correlation in the great majority of cases, but emphasizes his belief that some failures are due to an increased tolerance of the host to the drug, irrespective of the organism. In these few cases, the organism is in fact responsive to sulfonamides in vitro, and he reports successful clinical response after switching to another sulfonamide to which the host presumably has not acquired a tolerance. (See discussion: practical application.)

In the work reported here, our aim has been:

(1) To confirm the work mentioned above by an independent method.

(2) To provide the experimental basis for a rapid method of identifying sulfonamide-resistant strains with sufficient accuracy to permit prediction of the possible success of sulfonamide therapy in any given case.

The authors wish to express, at this point, their appreciation of the invaluable advice and guidance offered throughout this work by Dr. J. Howard Mueller of the Department of Bacteriology of Harvard Medical School, and by Dr. Oscar F. Cox, director of the G. U. clinic of the Boston dispensary, where the cases were studied.

\section{EXPERIMENTAL METHOD}

Pure cultures of gonococcus were isolated from male patients and incubated at partial tension on caseinhydrolysate-starch agar slants at $37^{\circ} \mathrm{C}$. (8).

Promptly at 18 hours, standard suspensions of the growths were made in buffered saline, ${ }^{2} \mathrm{pH} \mathrm{7.4,} \mathrm{to} \mathrm{match}$

2 Saline was used for this purpose because it was felt that greater accuracy could be obtained in preparing the standard suspensions than by the use of broth or serum. Because of the toxic effect of the saline, it is important to inoculate the plates immediately after the dilutions of the 


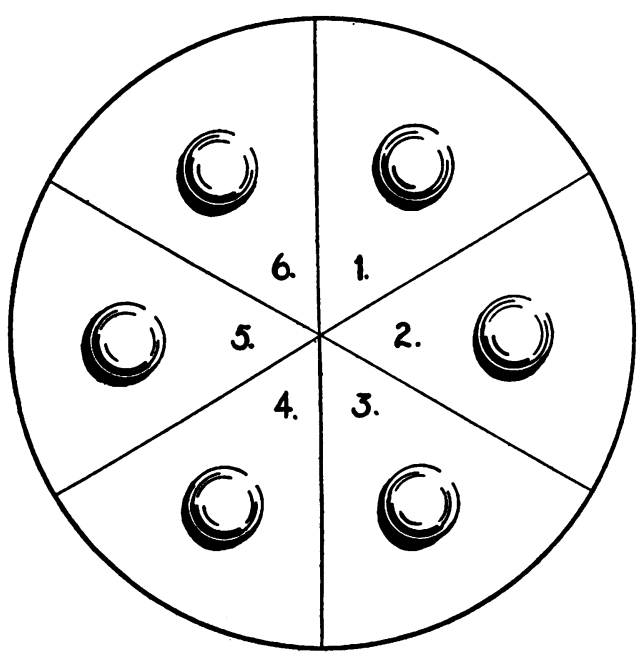

Fig. 1. Sketch of Inoculated Petri Plate

a McFarland suspension tube No. 1 under fluorescent lighting. Serial tenfold dilutions of $10^{-1}$ to $10^{-5}$ were then made in similar saline.

A drop of each of the 6 suspensions (standard and 5 dilutions) was placed in turn, each upon a marked sector of a starch agar petri plate, ${ }^{3}$ divided into 6 parts. Eight other plates were similarly inoculated, each of these plates containing varying amounts of sulfonamides as follows:

Plate No. 1 contained no drug,-Control

Plate No. 2 contained sulfathiazole, 0.001 per cent

Plate No. 3 contained sulfathiazole, 0.003 per cent

Plate No. 4 contained sulfathiazole, 0.005 per cent

Plate No. 5 contained sulfathiazole, 0.010 per cent

Plate No. 6 contained sulfadiazine, 0.001 per cent

Plate No. 7 contained sulfadiazine, 0.003 per cent

Plate No. 8 contained sulfadiazine, 0.005 per cent

Plate No. 9 contained sulfadiazine, 0.010 per cent

The drops of inoculum, from a capillary pipette of approximately constant caliber, were merely placed upon the medium, as shown in Figure 1, rather than streaked. By so doing, a constant number of organisms covered an approximately constant area of medium.

The plates were then incubated rightside up to avoid running of the drops, and examined at 18 hours. Growth appeared as a solid circle within the discrete boundaries of the original drops of inoculum, as discrete colonies within

original suspension have been made. Careful buffering to pH of 7.4, checked by a potentiometer each time before using, kept this toxic effect down to a minimum.

3 The p-amino benzoic acid, usually added routinely to promote growth of gonococci isolated from patients receiving sulfonamides, was omitted from the test medium. It might also be mentioned that the success of the method depends in part upon the use of a highly reproducible medium such as Mueller and Hinton's starch agar, containing a minimum of p-aminobenzoic acid, as well as relatively constant amounts of growth-promoting and growth-inhibiting substances. the same region, or finally as a faint ring visible only by reflected light, indicating that some slight reproduction had occurred in the first few hours of incubation before bacteriostasis was complete. In sectors reported as negative on each plate, not even a ring was visible where the drop of inoculum had been placed, the agar appearing completely untouched.

The control plate showed an average of 50 colonies in the inoculum from the sixth dilution of organisms (conc. $10^{-5}$ ). Since such quantitation of the number of organisms in each suspension was easily repeatable by the method of standardization used, it was possible to express the results of sulfonamide inhibition in vitro as the product of two factors, both essential in estimating resistance to the drugs: (1) The concentration of drug in the medium, and (2) the concentration of viable organisms inoculated.

In vitro results were reported in two ways (Tables I and II, Figure 2):

I. Resistance index. This arbitrary figure is the sum of all the positive growth readings found on the 4 plates containing sulfadiazine or sulfathiazole. The value of 1 was given to each clearly visible drop of growth, while drops visible only by reflected light were given a value of 0.5 . By inspection of Figure 1, it will be apparent that this
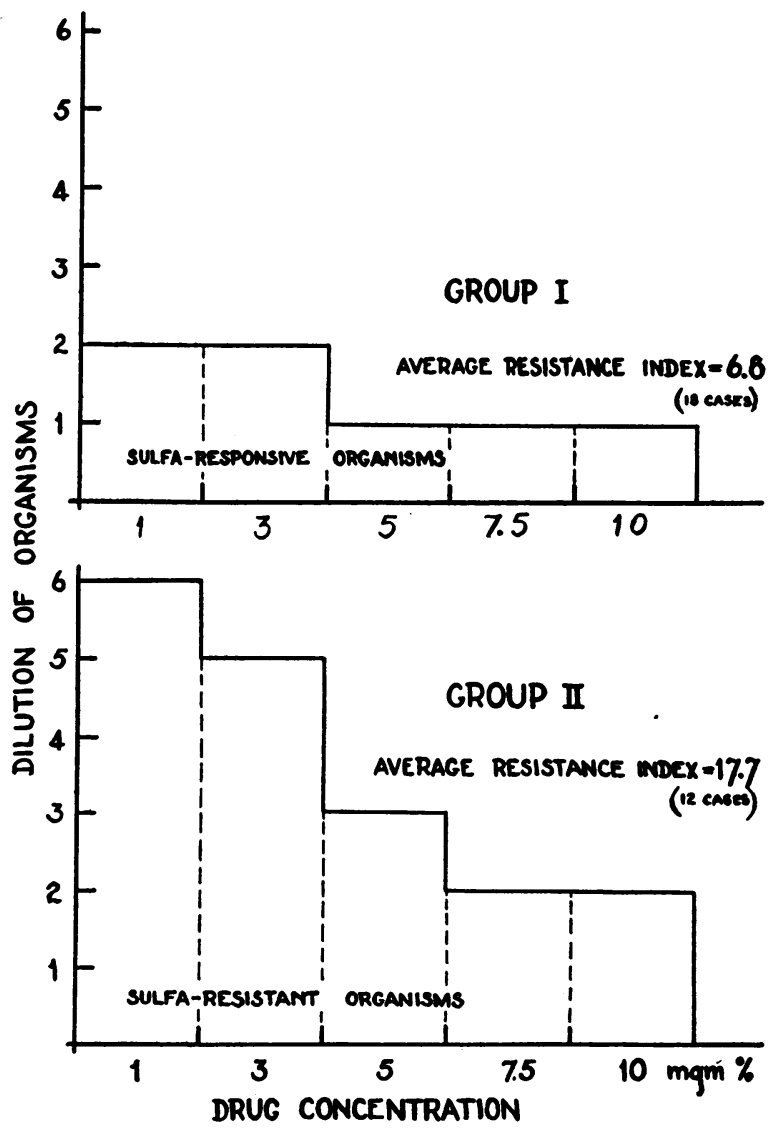

Fig. 2. Growth of Gonococci on SulfathlazoleContaining Media 
TABLE I

Individual case results

\begin{tabular}{|c|c|c|c|c|c|c|c|c|c|c|c|}
\hline \multirow{3}{*}{$\begin{array}{c}\text { Case } \\
\text { num- } \\
\text { ber }\end{array}$} & \multicolumn{2}{|c|}{ Clinical history } & \multirow{3}{*}{ Drug used* } & \multirow{3}{*}{$\begin{array}{l}\text { Days } \\
\text { after } \\
\text { onset } \\
\text { therapy } \\
\text { started }\end{array}$} & \multirow{2}{*}{\multicolumn{2}{|c|}{ Cure in }} & \multicolumn{4}{|c|}{ In vit } & \multirow{3}{*}{ Remarks } \\
\hline & \multirow{2}{*}{$\begin{array}{c}\text { Number of } \\
\text { previous Gc. } \\
\text { infections }\end{array}$} & \multirow{2}{*}{$\begin{array}{l}\text { Previous } \\
\text { sulfonamide } \\
\text { therapy }\end{array}$} & & & & & \multicolumn{2}{|c|}{$\begin{array}{c}\text { Resistance } \\
\text { index }\end{array}$} & \multicolumn{2}{|c|}{$\begin{array}{c}\text { Maximum } \\
\text { dilution }\end{array}$} & \\
\hline & & & & & Days & Visits & S.T. & S.D. & S.T. & S.D. & \\
\hline $\begin{array}{r}1 \\
2 \\
3 \\
4 \\
5 \\
6 \\
7 \\
8 \\
9 \\
10 \\
11 \\
12 \\
13 \\
14 \\
15 \\
16 \\
17 \\
18\end{array}$ & $\begin{array}{l}2 \\
? \\
2 \\
2 \\
0 \\
0 \\
0 \\
1 \\
2 \\
2 \\
0 \\
1 \\
0 \\
0 \\
2 \\
0 \\
1 \\
1\end{array}$ & $\begin{array}{l}0 \\
0 \\
? \\
0 \\
0 \\
0 \\
0 \\
0 \\
0 \\
0 \\
0 \\
0 \\
0 \\
\text { S.A.A. } \\
0 \\
0 \\
0 \\
0 \\
0\end{array}$ & $\begin{array}{l}\text { S.D. } \\
\text { S.D. } \\
\text { S.M. } \\
\text { S.M. } \\
\text { S.M. } \\
\text { S.D. } \\
\text { S.D. } \\
\text { S.T. } \\
\text { S.D. } \\
\text { S.M. } \\
\text { S.M. } \\
\text { S.D. } \\
\text { S.T. } \\
\text { S.D. } \\
\text { S.D. } \\
\text { S.M. } \\
\text { S.T. } \\
\text { S.M. }\end{array}$ & $\begin{array}{c}4 \\
1 \\
? \\
15 \\
5 \\
30+ \\
7 \\
0 \\
4 \\
6 \\
2 \\
3 \\
1 \\
23 \\
? \\
1 \\
2 \\
18\end{array}$ & $\begin{array}{l}0 \text { to } 3 \\
0 \text { to } 2 \\
0 \text { to } 5 \\
0 \text { to } 2 \\
0 \text { to } 6 \\
0 \text { to } 1 \\
0 \text { to } 1 \\
0 \text { to } 2 \\
1 \text { to } 5 \\
0 \text { to } 2 \\
1 \text { to } 3 \\
0 \text { to } 2 \\
0 \text { to } 2 \\
0 \text { to } 2 \\
1 \text { to } 2 \\
0 \text { to } 2 \\
0 \text { to } 1 \\
0 \text { to } 2\end{array}$ & $\begin{array}{l}1 \\
1 \\
1 \\
1 \\
1 \\
1 \\
1 \\
1 \\
2^{* *} \\
1 \\
2^{* *} \\
1 \\
1 \\
1 \\
2^{* *} \\
1 \\
1 \\
1\end{array}$ & $\begin{array}{l}6 \\
7 \\
5 \\
6 \\
5 \\
7 \\
7 \\
6 \\
7.5 \\
5 \\
8 \\
9 \\
7 \\
8 \\
8 \\
8 \\
6 \\
7\end{array}$ & $\begin{array}{r}5 \\
5 \\
5 \\
6 \\
6 \\
6 \\
7 \\
7 \\
7 \\
8 \\
8 \\
9 \\
9 \\
9 \\
9 \\
10 \\
10 \\
10\end{array}$ & $\begin{array}{l}2 \\
1.5 \\
1.5 \\
1 \\
1.5 \\
2.5 \\
2.5 \\
1.5 \\
2.5 \\
1.5 \\
2.5 \\
2.5 \\
2 \\
2.5 \\
2.5 \\
2.5 \\
1.5 \\
2.5\end{array}$ & $\begin{array}{l}1.5 \\
1.5 \\
1.5 \\
2 \\
2 \\
1.5 \\
2 \\
2 \\
2 \\
2 \\
3.5 \\
2.5 \\
3 \\
2.5 \\
3 \\
3 \\
3 \\
3\end{array}$ & $\left\{\begin{array}{l}\text { Complete cures after } \\
\text { first visit }\end{array}\right.$ \\
\hline $\begin{array}{l}19 \\
20\end{array}$ & $\begin{array}{l}? \\
1\end{array}$ & $\begin{array}{l}\mathbf{0} \\
\mathbf{0}\end{array}$ & $\begin{array}{l}\text { S.T. } \\
\text { S.D. }\end{array}$ & $\begin{array}{r}12 \\
4\end{array}$ & $\begin{array}{l}5 \text { to } 6 \\
6 \text { to } 8\end{array}$ & $\begin{array}{l}4 \\
4\end{array}$ & $\begin{array}{l}12 \\
16\end{array}$ & $\begin{array}{l}14 \\
16\end{array}$ & $\begin{array}{l}3.5 \\
4.5\end{array}$ & $\begin{array}{l}5.5 \\
4\end{array}$ & Intermediate \\
\hline $\begin{array}{l}21 \\
22 \\
23 \\
24 \\
25 \\
26 \\
27 \\
28 \\
29 \\
30 \\
31 \\
32\end{array}$ & $\begin{array}{l}0 \\
0 \\
2 \\
1 \\
0 \\
1 \\
2 \\
0 \\
0 \\
1 \\
1 \\
0\end{array}$ & $\begin{array}{l}\text { S.T., S.A. } \\
0 \\
\text { S.T. } \\
0 \\
? \\
0 \\
0 \\
0 \\
0 \\
\text { Yes (?) } \\
0 \\
\text { Yes (?) }\end{array}$ & $\begin{array}{l}\text { Local } \\
\text { S.M. } \\
\text { Local } \\
\text { S.D. } \\
\text { S.T., S.D. } \\
\text { S.D., S.T. } \\
\text { S.M. } \\
\text { S.D. } \\
\text { S.T., S.D. } \\
\text { S.D. } \\
\text { S.D. } \\
\text { Local }\end{array}$ & $\begin{array}{r}? \\
11 \\
? \\
2 \\
10 \\
1 \\
0 \\
? \\
0 \\
5 \\
3 \\
?\end{array}$ & $\begin{array}{l}90+ \\
26 \\
90+ \\
50+ \\
30+ \\
37 \\
38 \\
35 \\
150+ \\
? \\
30+ \\
30+\end{array}$ & $\begin{array}{l}6+ \\
6+ \\
6+ \\
6+ \\
6+ \\
6+ \\
6+ \\
6+ \\
6+ \\
\frac{6+}{6+}\end{array}$ & $\begin{array}{l}17 \\
16 \\
15 \\
18 \\
16 \\
18 \\
21 \\
13 \\
22 \\
19 \\
16 \\
15\end{array}$ & $\begin{array}{l}16 \\
17 \\
17 \\
19 \\
21 \\
22 \\
22 \\
23 \\
24 \\
24 \\
24 \\
24\end{array}$ & $\begin{array}{l}5 \\
6 \\
6 \\
6 \\
6 \\
6 \\
6 \\
5 \\
6 \\
6 \\
6 \\
6\end{array}$ & $\begin{array}{l}6 \\
6 \\
6 \\
6 \\
5 \\
6 \\
6 \\
6 \\
6 \\
6 \\
6 \\
6\end{array}$ & $\cdot$ \\
\hline
\end{tabular}

* $\mathrm{SA}=$ sulfanilamide, $\mathrm{SD}=$ sulfadiazine, $\mathrm{SM}=$ sulfamerizine, $\mathrm{ST}=$ sulfathiazole.

** The first of these 2 visits was less than 24 hours after starting therapy, at which time all showed negative smears, only slight discharge, and positive culture only in case \#11.

sum of positive readings expresses the ability of the organism to grow in the presence of a wide range of sulfonamide concentrations in all dilutions of the organism itself, thus the two vital factors in estimating sulfonamide resistance, mentioned above, have been accounted for by this value.

II. Maximum dilution. At 0.001 per cent of sulfadiazine, the sharpest differences between strains were observed, as measured by the maximum dilution of organisms at which growth occurred. This figure was therefore recorded to show how one single plate with this concentration of sulfadiazine could be used alone to test the resistance of strains under a simplified, more practical method (9).

\section{EXPERIMENTAL RESULTS}

Thirty-two cases of proven male gonorrhea were studied as outlined above. They fell into two main groups and one small intermediate group (of 2 cases), both on the basis of clinical

TABLE II

Summary of the average in vitro results

\begin{tabular}{ccc}
\hline \hline & $\begin{array}{c}\text { Resistance } \\
\text { Index }\end{array}$ & $\begin{array}{c}\text { Maximum } \\
\text { Dilution }\end{array}$ \\
\hline Group I-Responsive & 6.8 & 2 \\
Sulfathiazole in vitro & 7.6 & 2 \\
Sulfadiazine in vitro & 17.7 & 5.4 \\
Group II-Resistant & 21 & 5.7 \\
$\quad$ Sulfathiazole in vitro & & \\
$\quad$ Sulfadiazine in vitro & & \\
Group III-Intermediate & 14 & 4 \\
Sulfathiazole in vitro & 15 & 4.7 \\
Sulfadiazine in vitro & &
\end{tabular}

* Data from this group are insufficient for any definite conclusions. 


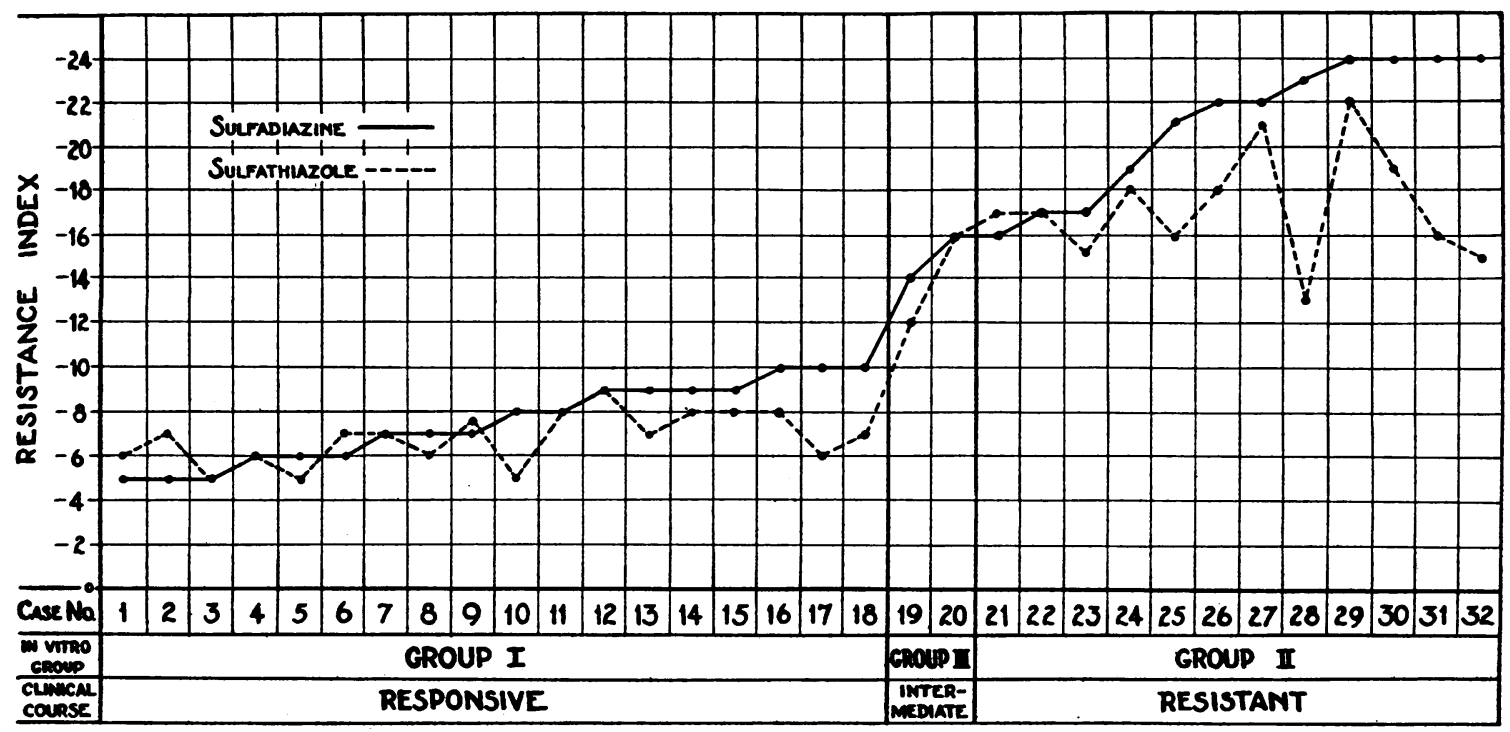

Fig. 3. Clinical and In Vitro Resistance of 32 Cases

response and the in vitro test. A strikingly close correlation was obtained in every case (See Table I and Figure 3).

Group I, consisting of 18 patients, showed negative smears and cultures, and were asymptomatic on the first examination after therapy was started, remaining negative at each subsequent follow-up examination. This first examination was usually made 24 to 48 hours after sulfonamide was initially administered, as shown in Table I, but in some cases, the first visit was made 3,5 , or 6 days after the beginning of treatment. All of the strains isolated from this group showed Resistance Indices of 5 to 9 with sulfathiazole and 5 to 10 with sulfadiazine. They grew out at most to only the third dilution of organisms inoculated on the plates containing the lowest concentration of each drug, $1 \mathrm{mgm}$. per cent.

Group $I I$, consisting of 12 patients, continued to show evidence of infection for 26 days or more; in other words, by Cox's criteria, they were true sulfonamide failures. Four of these cases had cultures taken for study before any sulfonamides were given, 4 were previous drug failures before admission to the clinic, and 4 were cultured at varying times after unsuccessful sulfonamide therapy in the dispensary. All of these 12 cases showed Resistance Indices varying from 13 to 24, and all grew out to a Maximum Dilution of 5 to 6 , at a concentration of $1 \mathrm{mgm}$. per cent of each drug.

Group III consisted of 2 cases, who were not cured after the first visit or first 48 hours of therapy, but who subsequently became completely negative, soon enough to attribute the cure to sulfonamides. One case was cured in 6 to 8 days, the other in 5 to 6 . In vitro, the 2 strains also gave results intermediate between the 2 groups, I and II, with Resistance Indices of 12 to 16, and Maximum Dilutions of 4 to 5.5 . This group is so small that one can only speculate on the presence of an intermediate mildly sulfonamide-resistant strain.

\section{DISCUSSION}

\section{Theoretical}

The results reported above confirm the correlation, noted by others, between the clinical course and the in vitro tolerance of the infecting gonococcus to sulfonamides. Such a complete correlation, with no exceptions found, constitutes positive evidence that sulfonamide resistance in male gonorrhea depends upon factors within the organism rather than upon factors within the infected host.

Additional evidence against any appreciable contribution to sulfonamide resistance by host factors follows, some of which throws light upon 
difficulties encountered in sulfonamide therapy of this disease:

(1) The uniformly short courses of the responsive group of cases (less than 2 days in all cases followed daily) are hard to explain except on a basis of absolute bacteriostasis by the drug alone. Host factors such as immune body titre could be regarded as important in determining the reaction of the patient to sulfonamide therapy only. if marked variations in the length of these courses were seen. It is also well known that gonococci prevented from reproducing will be destroyed rapidly by "autolysis," or by a rapid aging process. This may explain the rapid action of sulfonamides in responsive cases without aid from phagocytes and immune bodies.

(2) The rare intermediate type of clinical course showed positive signs of disease for about a week, followed by complete sulfonamide cure within 14 days. Even these cases, however, were infected with strains which showed a partial in vitro sulfonamide resistance compatible with the clinical picture and were best explained by variations within the organism rather than within the host.

Lankford et al. (6) have found that, under certain conditions, varying degrees of sulfonamide resistance, each of which can be estimated quantitatively, could be induced in vitro from strains previously found responsive to sulfathiazole.4 Such changes in the ability of the organism to tolerate the sulfonamides resembled in general the process of mutation, and the degree of resistance, once attained, remained constant. It is not unreasonable to suggest that a similar process may occur in nature, with varying degrees of sulfonamide resistance, perhaps acquired by mutation, accounting for several distinct clinical courses under sulfonamide therapy. In our small series, at least 2 variant strains, the immediately responsive and the completely resistant types, and possibly a third intermediate variant show up both clinically and in vitro. It is

- Landy et al. (10) and Stokinger, Charles, and Carpenter (11) have also induced sulfonamide resistance in vitro in formerly responsive strains. They identified an increase in the production of p-amino benzoic acid by the organisms with the acquisition of resistance, and offer this as a partial tentative explanation of the changes in metabolism involved. possible that other mutants with further variations in sulfonamide resistance also exist. It must be remembered, however, that as yet no demonstration has been made of a responsive strain becoming resistant in vivo under the influence of sulfonamide therapy in gonorrhea. Such a phenomenon has been demonstrated in cases of streptococcus viridans (12), pneumococcus endocarditis (13), and pneumococcus pneumonia (14).

(3) Regardless of the stage of the disease at which treatment is begun, sulfonamides seem to act promptly or not at all, leaving the course of a resistant infection unchanged as far as we can tell. This fact is obvious from Table $I$ in the column marked: "Days after onset when therapy was started." Case 14, for instance, received sulfanilamide for the first 2 weeks without effect and was first admitted to the clinic after 23 days of urethral discharge. He was found to have a positive culture, but to be infected with a strain susceptible in vitro to the more effective sulfonamides. His culture and symptoms became permanently negative within 24 hours of the institution of sulfadiazine therapy.

In other words, it appears that a responsive case will be cured by sulfonamides promptly, no matter how long symptoms have been present, while a resistant case will run its course, no matter how soon or how late the drug is started, or how many courses of sulfonamides are given, in the overwhelming majority of cases. ${ }^{5}$

\footnotetext{
- The rarity of success of a second course of sulfonamides where a first course has failed, as well as the dangers of inducing drug resistance in the host by insufficient dosage, are discussed by Cox (15), who above all would discourage the indiscriminate use of multiple courses of drug in resistant infections, especially if the organism proves to be drug resistant in vitro as well.

The rare prompt response actually attributable to a subsequent course of chemotherapy in an initially resistant infection, as studied in the Boston Dispensary, usually occurs in already waning infections; these cases usually have persisted for over 4 weeks, with subsidence of acute symptoms, and signs that the natural process of selflimitation of the disease is already underway. Adequate in vitro studies on such cases are not complete yet, but we believe that one of several circumstances may account for the success of a second course of sulfonamides where a first has failed:

(1) Attenuation of the organism may occur in the presence of an increasingly hostile and immune host. Such
} 
The clinical picture of the resistant infections is therefore essentially the same as the disease seen before the days of sulfonamide therapy-in our series, infections lasting from 26 days to many months. In these cases, excluding the rare partially resistant infections, there is no evidence that sulfonamides have any appreciable therapeutic effect, by the criteria of Cox (1). Within this resistant group it is therefore logical that host factors may well help to determine whether a man will be free from disease within a few weeks or within a matter of months.

The essential fact remains, however, that whether or not the initial and prompt cure with sulfonamides will be obtained at all seems to depend upon a contest between organism and drug in a relatively neutral host.

\section{Practical application}

In all 32 cases studied, it was possible to predict the clinical course that would follow treatment with sulfonamides. It was found that a "typing" of the organisms into responsive and resistant groups could be obtained with accuracy by the use of one plate containing $1 \mathrm{mgm}$. per cent of each sulfonamide, indicating that considerable simplification of the method would be possible. (The details of a simplified method have already been worked out and published, and it is now being used routinely in the $G$. U. clinic of the Boston Dispensary under the direction of Dr. Cox (9).)

Recent work (2) has shown that infections may show marked variations in response depending upon whether sulfathiazole, sulfadiazine, or sulfamerizine is used. These cases are not common, but are brought more forcibly to one's attention by the fact that the variations appear corre-

a change might appear as a decrease in the resistance to sulfonamides of the organism subsequently cultured from the patient. As yet, however, there are no reports of a resistant strain changing to a responsive variant in the host.

(2) A different drug may have been used for the late course, the potency of which was greater against the particular organism involved than that of the original drug. (See discussion, Practical application.)

(3) Host immunity may have arisen to the point at which slight aid from previously ineffective sulfonamides will complete the elimination of the infection.

(4) Insufficient dosage may have been used initially. spondingly in vitro, as illustrated by the following case:

B. D. No. 427038 entered the clinic with a fresh untreated infection. The organism cultured before starting chemotherapy was typed in vitro as resistant to sulfamerizine but responsive to sulfathiazole. Meanwhile, through misunderstanding, sulfamerizine was administered to the patient, without response. After 16 days of persisting resistant infection, a course of sulfathiazole was started, with prompt and permanent response. Both the resistance to sulfamerizine and the response to sulfathiazole were thus predicted by the original in vitro typing on admission.

It is therefore wholly logical that cases should occasionally appear to be completely resistant to therapy with one drug and later respond perfectly to a second course using another drug which, in this particular infection, happens to be more effective, both clinically and in vitro. Harkness (7), on the other hand, believes that variations in response to different sulfonamides are due to variations in the host's tolerance for the drug. Although his explanation may also hold true in a small additional percentage, variations in potency of the several sulfonamides seem to explain adequately those cases studied in the Boston Dispensary.

The ability to predict the results of sulfonamide therapy by the use of this typing method has several obvious advantages:

(1) If the organism is responsive in vitro, the patient can be discharged safely after a short course of sulfathiazole, sulfadiazine, or sulfamerizine, without risk of relapse, and can remain ambulatory throughout treatment. The most effective of the available drugs indicated should also be determined and administered accordingly, by typing the organism on plates containing each of the drugs available. ${ }^{6}$

(2) The finding of an organism resistant to a sulfonamide in vitro will immediately indicate that the patient will probably not respond well,

\footnotetext{
- In most cases, sulfathiazole, sulfadiazine, and sulfamerizine seem to be equally effective (or ineffective) in gonorrhea, both in vitro and in vivo. In the borderline cases, where differences in potency show up, sulfamerizine is definitely weaker, and sulfadiazine very slightly weaker, than sulfathiazole. Inexplicable exceptions to this order of potency occasionally appear. Sulfapyridine is of consistently lower potency, while sulfanilamide is comparatively ineffective.
} 
if at all, to that sulfonamide. If the organism is resistant in vitro to all available sulfonamides, then another form of therapy is advisable. Where facilities are available, such patients might often be cured promptly by the use of fever therapy or penicillin $(16,17)$, both of which are effective in a high percentage of sulfonamideresistant cases. Also, repeated courses of sulfonamides should not be used in these cases where chances of success are slim, and where chances for toxic effects and sensitivity formation become increasingly great.

(3) Finally, once a patient has failed to respond, as in Case 14, the chances of success with a second course of the same or a different drug can be determined by retyping the organisms grown out on subsequent cultures.

\section{SUMMARY}

(1) Thirty-two cases of male gonorrhea, treated with sulfonamides, have been analyzed.

(2) Correlation between clinical and in vitro resistance of the infecting strain to sulfathiazole and sulfadiazine has been demonstrated in every case studied.

(3) This correlation indicates that those factors within the gonococcus which determine in vitro resistance to sulfonamides also determine whether the infection will respond promptly and permanently to sulfonamides, or show varying degrees of clinical resistance.

(4) Host factors appear to be relatively unimportant, except in those infections in which the course is initially unaffected by drug therapy.

(5) The in vitro resistance of the organism, and hence the clinical response to be expected under sulfonamide therapy, can be accurately estimated by the method outlined.

(6) The clinical value of a simplified modification of this "sulfonamide resistance typing" technique has been presented. It is suggested that such in vitro methods have a possible application in other bacterial infections, placing the use of bacteriostatic drugs on a more rational basis, as well as discouraging the use of futile indescriminate courses of sulfonamides where the organism is obviously resistant.

\section{REFERENCES}

1. Cox, O. F., Chemotherapy in gonococcal infections. New England J. Med., 1942, 226, 184.

2. Cox, O. F., Unpublished data.

3. Petro, J., Sulfonamide resistance in gonorrhea. Lancet, 1943, 1, 35.

4. Cohn, A., Steer, A., and Seijo, I., Correlation between clinical and in vitro reactions of gonococcus strains to sulfathiazole. Am. J. M. Sc., 1942, 203, 276.

5. Bang, F. B., and Bang, B., Sulfanilamide, sulfapyridine, and sulfathiazole therapy of gonococcal infection of the chorio-allantoic membrane. Proc. Soc. Exper. Biol. and Med., 1941, 46, 527.

6. Lankford, C. E., Scott, V., and Cooke, W. R., Studies of sulfonamide resistance of the gonococcus. J. Bact., 1943, 45, 201.

7. Harkness, A. H., Sulphonamide resistance. Lancet, 1943, 2, 116.

8. Mueller, J. H., and Hinton, J., A protein-free medium for primary isolation of the gonococcus and meningococcus. Proc. Soc. Exper. Biol. and Med., 1941, 48, 330.

9. Goodale, W. T., Gould, R. G., Schwab, L., and Winter, V., Laboratory identification of sulfonamide resistant gonococcic infections. J. A. M. A., 1943, $123,547$.

10. Landy, M., Larkum, N. W., Oswald, E. J., and Streightoff, F., Increased synthesis of p-aminobenzoic acid associated with the development of sulfonamide resistance in staphylococcus aureus. Science, 1943, 97, 265.

11. Stokinger, H. E., Charles, P. C., and Carpenter, C. M., The role of p-aminobenzoate in experimentally developed sulfanilamide-fastness in the gonococcus. J. Bact., 1942, 44, 261.

12. Janeway, C. A., and Schwachmann, A., Personal communication.

13. Hamburger, M., Jr., et al., Sulfonamide resistance developing during treatment of pneumococcic endocarditis. J. A. M. A., 1942, 119, 409.

14. Lowell, F. C., Strauss, E., and Finland, M., Observations on the susceptibility of pneumococci to sulfapyridine, sulfathiazole and sulfamethylthiazole. Ann. Int. Med., 1940, 14, 1001.

15. Cox, O. F., Sulfonamide-resistant gonococcal infections. Bull. Genitoinfect. Dis., 1943, 6, 1-3.

16. Herrell, W. E., Cook, E. N., and Thompson, L., Use of penicillin in sulfonamide resistant gonorrheal infections. J. A. M. A., 1943, 122, 289.

17. Keefer, C. S., et al., Penicillin in the treatment of infections. J. A. M. A., 1943, 122, 1217. 